Document downloaded from:

http://hdl.handle.net/10251/64686

This paper must be cited as:

Pernice, R.; Parisi, A.; Ando, A.; Mangione, S.; Garbo, G.; Busacca, AC.; Perez, J.... (2015). Error mitigation using RaptorQ codes in an experimental indoor free space optical link under the influence of turbulence. IET Communications. 9(14):1800-1806. doi:10.1049/ietcom.2015.0235.

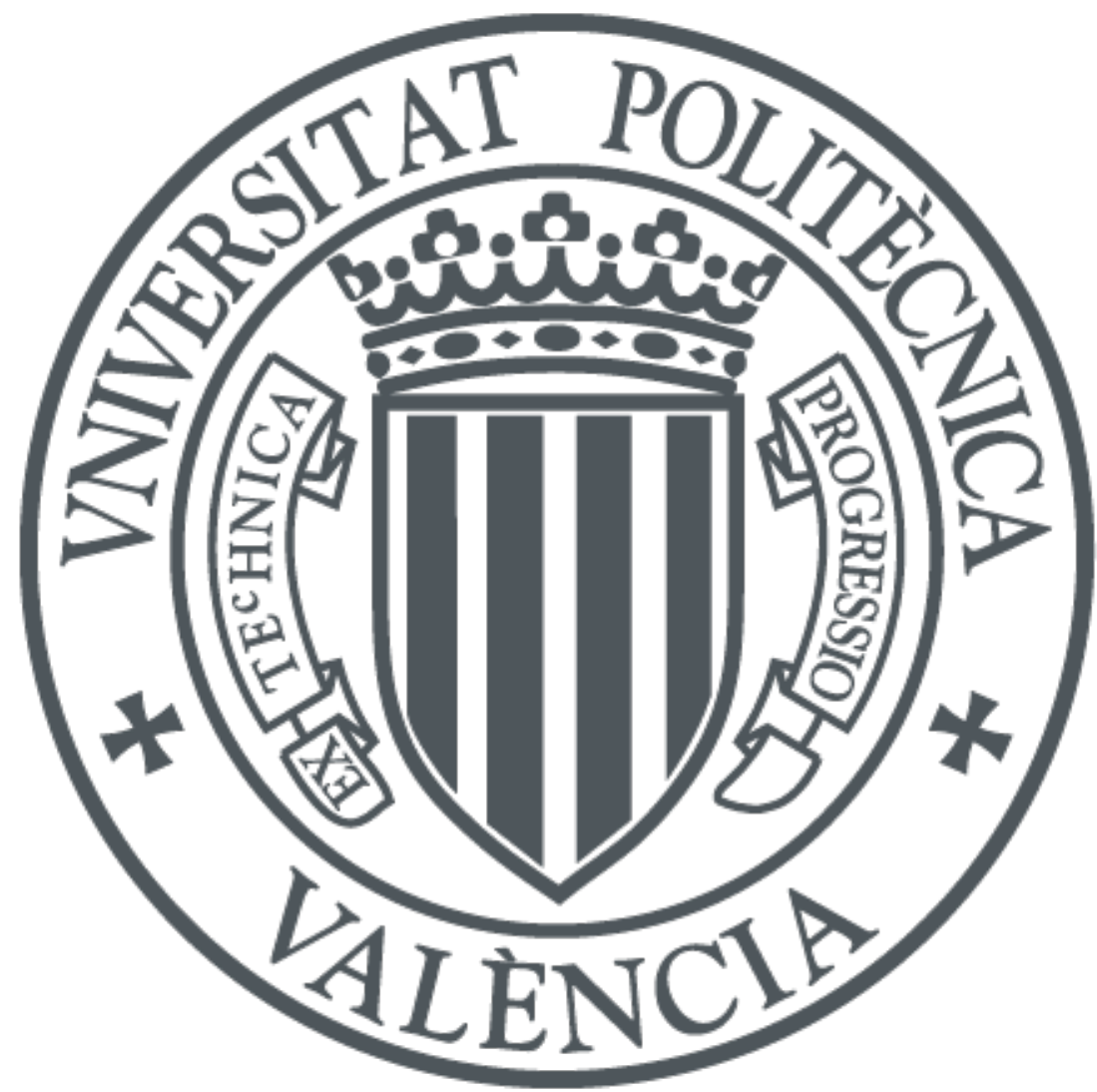

The final publication is available at

http://dx.doi.org/10.1049/iet-com.2015.0235

Copyright Institution of Engineering and Technology (IET)

Additional Information

This paper is a postprint of a paper submitted to and accepted for publication in [journal] and is subject to Institution of Engineering and Technology Copyright. The copy of record is available at IET Digital Library 


\title{
Error mitigation using RaptorQ codes in an experimental indoor FSO link under the influence of turbulence
}

\author{
R. Pernice ${ }^{1}$, A. Parisi ${ }^{1}$, A. Andò ${ }^{1}$, S. Mangione ${ }^{1}$, G. Garbo ${ }^{1}$ and A.C. Busacca ${ }^{1}$ \\ J. Perez ${ }^{2}$, \\ Z. Ghassemlooy ${ }^{3}$ \\ 1 Department of Energy, Information engineering and Mathematical models (DEIM) \\ University of Palermo, 90128, Palermo, Italy \\ 2 Optical and Quantum Communications Group, Institute of Telecommunications and Multimedia Applications \\ (iTEAM), Universitat Politècnica de València, 46022, València, Spain \\ 3 Optical Communications Research Group, NCRLab, Faculty of Engineering and Environment, Northumbria \\ University, Newcastle upon Tyne, NE1 8ST, United Kingdom
}

Correspondence should be addressed to Riccardo Pernice; riccardo.pernice@ unipa.it

\begin{abstract}
In Free space optical (FSO) communications, several factors can strongly affect the link quality. Among them, one of the most important impairment that can degrade the FSO link quality and its reliability - even under the clear sky conditions - consists of optical turbulence. In this work, we investigate the generation of both weak and moderate turbulence regimes in an indoor environment to assess the FSO link quality. In particular, we show that, due to the presence of the turbulence, the link experiences both erasure errors and packet losses during transmission, and also compare the experimental statistical distribution of samples with the predicted Gamma-Gamma model. Furthermore, we demonstrate that the application of the RaptorQ codes noticeably improves the link quality decreasing the packet error rate (PER) by about an order of magnitude, also offering - in certain cases - an error free transmission with a PER of $\sim 10^{-2}$ at Rytov variance value of 0.5 . The results show that the recovery rate increases with the redundancy, the packet length and the number of source packets, while decreases with increasing data rates.
\end{abstract}

\section{Introduction}

Free space optics (FSO) based technologies offer a number of noteworthy advantages over the traditional radio frequency (RF) counterparts including increased security, higher bandwidth, easy installation and deployment of the equipment, low power consumption, and immunity to the electromagnetic interference $[1,2]$. Commercial FSO systems can operate at data rates $R$ up to $10 \mathrm{Gbps}[3,4]$ over transmission span of a few kilometers. The data rate can be further increased by employing the wavelength division multiplexing (WDM) technique reaching 1.6 Tbps over a short link span of couple hundred meters [5, 6]. FSO links are being used for both satellite and terrestrial applications and also finding their ways into indoor environment. For example, ground-to-ground short-to-medium range links in rural areas, where deployment cost is still high for the end users, are a solution to the 'last mile' access network bottleneck [1]. Moreover, they are largely 
being employed as temporary, back-up and disaster recovery links, in inter-satellite communications and/or satellites-toearth links $[7,8]$.

However, FSO links implementation contemplates challenges such as the maintenance of the line-of-sight condition between the transmitter and the receiver during the transmission, in particular for the case where the optical beams footprint at the receiver is comparable to the photodetector surface area. In fact, temporary misalignments, for example due to pointing errors, could lead to data loss or even interrupt the transmission. Additionally, the propagating optical beam can also be severely affected by the adverse atmospheric conditions (i.e., rain, fog, snow, smog - that generate Rayleigh and Mie scattering - and turbulence [9]). Turbulence, also known as the scintillation, is almost entirely generated by the small temperature variations in the atmosphere, which results in refractive index fluctuations along the optical propagation path $[10,11]$. Thus leading to the power loss and irradiance fluctuations (fading), beam wondering and beam spreading at the receiver that can result in increased bit error rate (BER), decreased channel capacity and link reliability when the optical power level drops below a fading depth (i.e. a threshold below which the receiver is not able to detect the signal) [12]. Moreover, deep power fades may also lead to severe communication outages.

A number of solutions, both hardware - such as the spatial diversity, e.g., multiple-input multiple-output (MIMO) FSO systems [1, 13, 14], adaptive optics [15], and large receiver aperture sizes [1] - and software (i.e. error control coding, channel coding) have been reported in the literature to mitigate atmospheric turbulence-induced fading in intensity modulation/direct detection (IM/DD) FSO links. In digital communications channel coding is essential to approach the channel capacity- as predicted by the information theory - and to achieve reliable information transmission. FSO systems can be assumed to be slowly time-variant since the received optical power variation in time is in the order of milliseconds while the data (symbol) channels are in the order of Mbps or Gbps [1]. In FSO systems most uncoded bit errors occur during channel fades, because of the burst-error channel condition. The Reed-Solomon (RS) codes have been used as channel error correcting codes [16]. In [17], space-time block coding for MIMO based FSO systems were proposed. The repetition coding and transmit laser selection schemes have been proposed for FSO links where the path gains are real and non-negative $[18,19]$.

The rateless codes $(\mathrm{RC})$ are the most recent software based solution [20-29], specifically designed for the erasure channels because of ease of operation and no rate mismatch as a result of imprecise channel estimation. RC have high potential for increasing the capacity of wireless systems in which the information is transmitted at a very high $R$ and at the receiver it is accumulated in a form of graph, trellis, or matrix structure. In RC a sequence of encoding symbols could be produced from a given source symbols (blocks) with added redundancy during the transmission. Subsequently, at the receiver the original source block can be successfully recovered with high probability from any subset of the encoding symbols with equal or slightly larger size than the number of source symbols. Compared to other codes, RC has not been widely adopted in FSO systems. In [30] the information rates of RC and punctured LDPC codes with feedbacks were 
compared for FSO links. However, the evaluation of the codes was carried out offline using the recorded measured data. In this paper, we experimentally investigate the use of most recent and powerful RC (i.e., RaptorQ codes (RQC) [25-29]) for an FSO system and compare the link performance under both weak and moderate turbulence regimes using an indoor experimental set up.

In detail, we describe an indoor FSO communications link under turbulence regimes within a controlled environment. In order to demonstrate the effectiveness of our system, we compare the statistical distribution of the samples recorded during the measurements with the most widely used theoretical distribution (i.e., the Gamma-Gamma (GG) model, which describes all turbulence regimes $[1,10,11])$. We demonstrate how the packet error rate (PER) decreases by recovering most of the lost packets. Hence, we can conclude that RaptorQ codes, if configured appropriately, represent an alternative viable solution to reduce the number of errors in FSO links under turbulence conditions.

\section{Turbulence Model}

Several mathematical models have been reported in the literature to predict the impact of turbulence on the FSO link performance in the way of analyzing and assessing the optical irradiance fluctuations at the receiver [10, 11]. Among them, lognormal model [4] has been adopted for the weak turbulence regime, while for the strong turbulence regime (i.e. the saturation regime) the negative exponential model has been used. However, the most versatile and widely used model is GG that defines all turbulence regimes [31], which has been adopted in this work.

For FSO links the channel model can be represented as:

$$
y=h_{c} x+n
$$

where $y$ is the received signal at the detector, $h_{c}$ is the channel gain, $x$ is the transmit signal intensity, and $n$ is the additive white Gaussian noise that zero mean and variance $\sigma_{n}{ }^{2}$. Under the atmospheric turbulence channel condition the scintillation index, which is the metric for measuring the normalized intensity variance, is defined as $[4,32]$ :

$$
\sigma_{I}^{2}=\frac{\left\langle I^{2}\right\rangle}{\langle I\rangle^{2}}-1
$$

where $I$ is the irradiance of the optical wave and the angle brackets $<>$ represent an ensemble average. For the GG model, we assume that both large-scale and small-scale irradiance fluctuations (i.e., independent random process) are governed by the Gamma distributions as outlined in [10]. The probability density function (PDF) of the received optical irradiance can be expressed in terms of independent random process with Gamma distribution as [10, 11]:

$$
p(I)=\frac{2(\alpha \beta)^{(\alpha+\beta) / 2}}{\Gamma(\alpha) \Gamma(\beta)} I^{\{[(\alpha+\beta) / 2]-1\}} K_{\alpha-\beta}(2 \sqrt{\alpha \beta I})
$$


where $\Gamma(\cdot)$ is the Gamma function, and $K_{n}\left({ }^{\cdot}\right)$ is the modified Bessel function of the second kind of order $n . \alpha$ and $\beta$ are two parameters, which are related to the large-scale and small-scale scintillations of the optical wave, respectively, and are given by $[10,11]$

$$
\begin{aligned}
& \alpha=\left[\exp \left(\sigma_{\ln x}^{2}\right)-1\right]^{-1} \\
& \beta=\left[\exp \left(\sigma_{\ln y}^{2}\right)-1\right]^{-1}
\end{aligned}
$$

$\sigma_{l n x}^{2}$ and $\sigma_{l y x}^{2}$ are the large scale and the small scale log-irradiance variances, respectively, and are defined, for the plane wave model at the zero inner scale case, as follows [10,11]:

$$
\begin{aligned}
& \sigma_{\ln x}^{2} \cong \frac{0.49 \sigma_{R}^{2}}{\left(1+0.65 d^{2}+1.11 \sigma_{R}^{12 / 5}\right)^{7 / 6}} \\
& \sigma_{\ln y}^{2} \cong \frac{0.51 \sigma_{R}^{2}\left(1+0.69 \sigma_{R}^{12 / 5}\right)^{-5 / 6}}{1+0.90 d^{2}+0.62 d^{2} \sigma_{R}^{12 / 5}}
\end{aligned}
$$

where $d$ is a scalar parameter given by $[10,11]$ :

$$
d=\sqrt{k D^{2} / 4 L}
$$

where $k$ is the wavenumber, $D$ is the receiver diameter and $L$ is the propagation path length.

The scintillation index can be also derived from $\sigma_{\ln x}^{2}$ and $\sigma_{\ln y}^{2}[10,11]$ :

$$
\sigma_{I}^{2}=\exp \left(\sigma_{\ln x}^{2}+\sigma_{\ln y}^{2}\right)-1
$$

and Rytov variance $\sigma_{R}^{2}$ can be determined considering the expressions (6) and (7) and thus inverting equation (9) and given as:

$$
\sigma_{R}^{2}=1.23 S k^{7 / 6} L^{11 / 6}
$$

where $S$ is the structural parameters of refractive index. The turbulence regimes for weak, moderate and strong are defined in terms of Rytov variance of $\sigma_{R}^{2} \leq 0.3,0.3<\sigma_{R}^{2} \leq 5, \sigma_{R}^{2}>5$, respectively [31].

\section{Rateless Codes}

Several software techniques have been used to reduce the errors occurring during data communication. In particular, in an erasure channel, where a transmitted packet is either received without error or completely lost [22], a widely employed technique makes use of a retransmission protocol. In this way, the receiver sends back messages that identify the erased packets, which are then retransmitted by the sender. However, this method is often not convenient, since both the feedback and the retransmitted packets are redundant information, thus resulting in reduced overall transmission throughput. 
Moreover, retransmission is inappropriate or even cannot be used for multicast and/or broadcast applications (e.g., video on demand, and digital TV), in which the information is transmitted from a single source to multiple receivers, as each receiver can lose different portions of the original message. Also, in audio/video streaming and conference applications lost data will cause a decrease in the link quality, therefore these services cannot benefit from retransmission due to realtime requirements. In the above-mentioned cases, retransmission of the lost packets would then be intolerably redundant. For this reason, forward error correction (FEC) codes, with no feedback packets, such as Reed-Solomon codes have been proposed $[21,23]$.

A better solution would be to use RC, which have been designed for erasure channels [22]. RC are also known as "fountain codes" (FCs), since the encoder can be thought as a "fountain" producing, theoretically, endless supply of "water drops" (i.e., packets), in other words an infinite amount of redundancy being added to the information bits of a source. The transmitter divides the file of length $L_{d}$ into a number of $K=L_{d} / L_{p}$ packets where $L_{p}$ is packet length, and adds a number of $K_{r}$ redundant packets. The receiver would be able to recover the original data, provided the received number of packets is slightly larger than $K$. Fountain codes are rateless, since the transmitter can change the number of generated encoded packets on the fly, according to the channel conditions, and this is the main strength of this class of codes compared to traditional FEC schemes [21, 23].

The working principle of random linear fountain codes is described in detail in [22]. Herein, we only underline the main features of these codes, and also of Luby Transform (LT), Raptor and RQC, which represent the most recent rateless codes available today. In random linear FCs, for each group of $K$-packet a new set of $N$ encoded packets $(N>K)$ is generated, and a linear combination (bitwise sum, modulo-2) of the $K$-source packets is performed by means of a binary pseudorandom G matrix $(K \times N)$ [20]. Each encoded packet has a number of $\delta$-link to the original source packets $(\delta$ is called "degree"). Assuming that the receiver will collect $N_{r}$-packet, then we will have the following three different scenarios [22]:

- If $N_{r}<K$ then the receiver will not have sufficient information to be able to recover the original data;

- If $N_{r}=K$ then the decoding process can start with the recovery probability of 0.289 for any $K>10$;

- If $N_{r}>K$ (and in particular for $N_{r}=K+h$, where $h$ is a small number of excess packets), then the probability of correctly decoding would be equal to $1-2^{-h}$.

An important limit of these codes is that their encoding and decoding costs are quadratic and cubic in the number of encoded packets, respectively. Hence, they are suitable only if $K$ is small (approximately < 1000).

LT codes show an improved performance by reducing the encoding and decoding complexities and decreasing the failure probability $[21,22]$. LT codes employ a robust soliton distribution, which guarantees a higher probability to complete the decoding process, at the same overhead, compared to the ideal soliton distribution [19, 21]. Moreover, the encoding and decoding costs scale as $K \log _{\mathrm{e}} K$, which is lower than the random linear FCs. 
RC was introduced by Shokrollahi, which represents the evolution of LT codes [23, 24]. The basic idea behind RC is to perform encoding/decoding process in two separate phases: (i) a pre-coding of the input symbols and (ii) the subsequent application of an appropriate weakened LT code. The chosen pre-coding scheme consists of a low-density parity-check (LDPC) code, whereas the selected LT code has an average degree $\delta$ of 3. In this way, RC achieve linear encoding and decoding. Moreover, contrary to LT codes, they are systematic - i.e., codes in which the first $K$-encoded packets are the original source packets - and the last packets are the redundant ones produced by the encoding process. An important advantage of systematic codes is that when there are no packet losses during the transmission, the receiver will directly collect all $K$-source packets in the correct order, without the need for decoding. In addition, it is worth noting that RC work on Galois Field GF(2) (i.e., XOR operations), thus ensuring that the probability of correctly perform decoding is at least 1$2^{-(h+1)}$ if $(K+h)$-packet are collected at the receiver. Moreover, the maximum value for $K$ is 8192 , while up to 65535 encoded packets can be generated during the encoding process [25].

Finally, the next generation of RC - known as RQCs - has been introduced [25-27]. They are one of the most advanced FEC codes for data networks offering improved coding performance in terms of reduced packet overhead for guaranteeing successful decoding, and supporting larger source packets block sizes. RQCs are systematic (as RC), and employ a RQ encoder that cascades two sub-encoders that employ both LDPC and high density parity check (HDPC) codes. In addition, some operations are based on the Galois Field (GF) (256). However, this kind of operations requires a higher computational cost when compared to XOR operations. For this reason, RQCs use a clever combination of GF(256) and GF(2) operations, in order to obtain the best recovery capabilities without increasing too much the computational cost. In particular, the majority of operations would be over GF(2), while the minority is over GF(256). In this way, when $(K+h)$ packets are collected by the RQ decoder, the probabilities of successful decoding are equal to $99 \%, 99.99 \%$, and $99.9999 \%$ for $h=0, h=1$, and $h=2$, respectively, and so on [27]. Finally, it is worth noting that RQCs work on a much larger alphabet set, thus supporting a maximum $K$ of 56403 source packets and up to 16777216 encoded packets.

\section{Experimental Set-up}

The schematic block diagram of the indoor experimental set-up is illustrated in Fig. 1. We have used two 100TX-to100FX electrical-to-optical (and vice versa) media converters MC1 and MC2 with input (Rx) and output (Tx) ports and operating at a wavelength of $1310 \mathrm{~nm}$. Rx1 is connected to Tx2 via a $5 \mathrm{~m}$ long multi-mode optical fiber (MMF), which is used as a feedback link between Rx1 and Tx2, while Rx2 is connected to the Tx1 port of MC1 via two different $1 \mathrm{~m}$ long MMFs and a 2.5 m-long FSO link. An optical collimator (OC) is used to launch the optical beam into the free space channel. To create a thermal gradient and hence to generate the turbulence we have used two $25 \mathrm{~cm}-$ long heating elements along the optical beam propagation path (OBPP) $[10,11]$. In $[29,33,34]$ it has been experimentally demonstrated that turbulence can be generated over a short FSO link. A fan placed close to the collimator is used to generate the air flux 
towards the heating elements, in order to create more controlled and repeatable turbulence conditions. An optical attenuator located just after OC is used to reduce the optical intensity in order to diminish the mean value received by MC2, thus allowing packet losses even under a very weak turbulence regime.

In order to accurately monitor and measure the temperature profile along the OBPP, we have used three fiber-optics thermometers (model Nortech Reflex) (T1, T2 and T3, see Fig. 1). T1 and T3 are placed above the two heating elements, while $\mathrm{T} 2$ is positioned between them. Using the heating elements, we have managed to achieve a maximum thermal gradient of $125^{\circ} \mathrm{C}$.

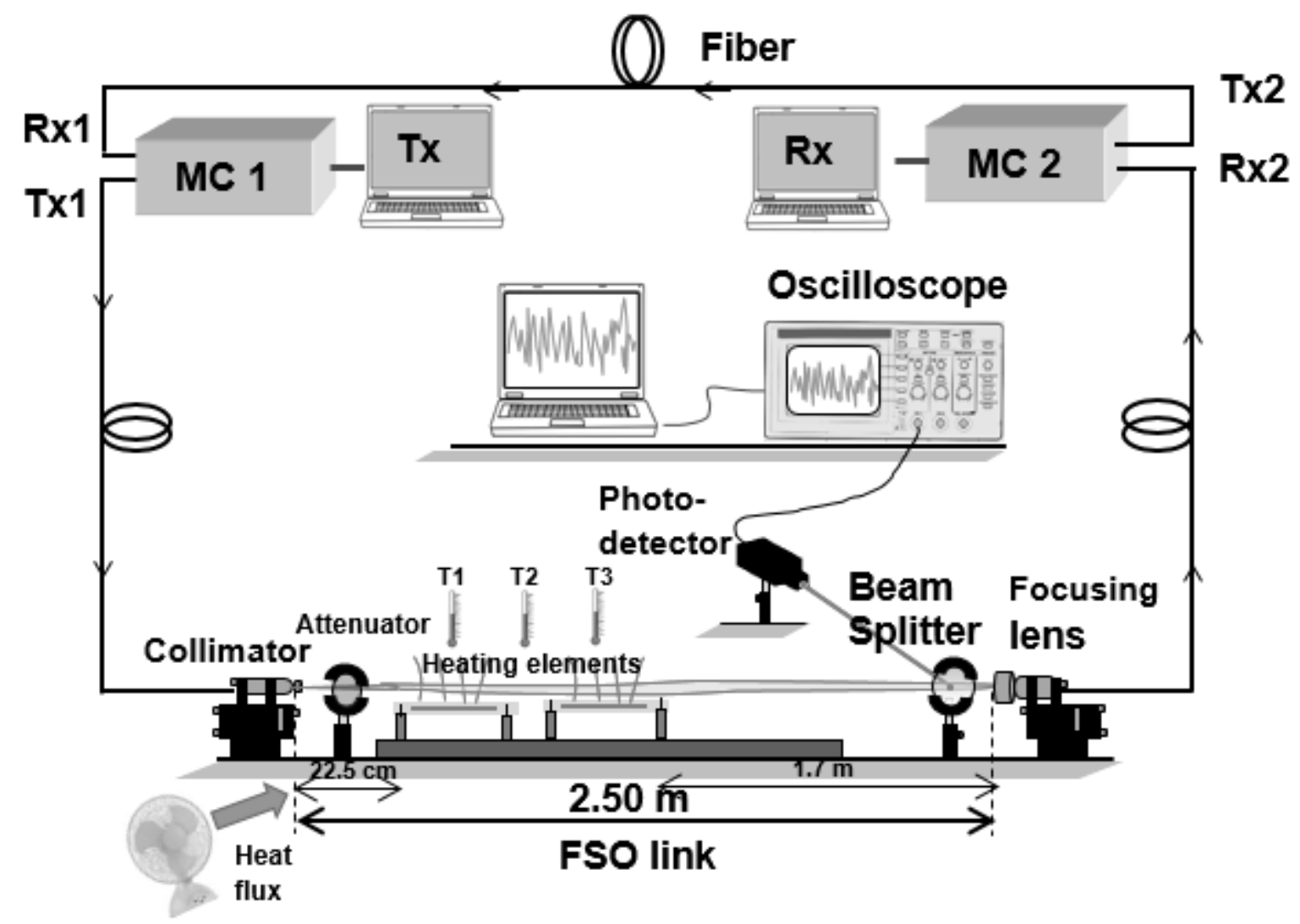

FIG. 1. Schematic block diagram of the experimental set-up.

At the receiving side of the FSO link, a beam splitter (splitting ratio of $90 / 10$ ) is used to capture a portion of the optical beam using a PD (active area of $0.38 \mathrm{~cm}^{2}$ ) for further real-time signal processing by means of a wide bandwidth $(350 \mathrm{MHz}$ and 2GSa/s sampling rate) digital storage oscilloscope (DSO). A focusing lens (having an active area of $0.38 \mathrm{~cm}^{2}$ ) is used to launch the optical beam into a MMF which is connected to $\mathrm{Rx} 2$ port of MC2. Data streams are generated using personal computers (PC) connected to MCs via Ethernet cables. Installed in both PCs is a dedicated developed file transmission software, which integrates RQC, implemented according to RFC 6330 [26]. Using the software we generate a number of encoded matrices, each composed of $K$-source and $K_{r}$-redundant packets. Each packet is composed of $\left(L_{p}+5\right)$-byte, where the additional 5-byte represents the encoding symbol ID (ESI) (i.e. information that uniquely identifies each encoding symbol). Using the file transmission software it is both possible to easily change the encoding/decoding parameters 
according to the channel conditions, as well as being able to evaluate in real time the packet losses and the packet recovery rate, as will be discussed in the next section.

The main parameters of the experimental FSO system are summarized in Table I.

TABLE I: KEY SYSTEM PARAMETERS

\begin{tabular}{|c|c|}
\hline Parameters & Values \\
\hline \multicolumn{2}{|l|}{ Media converters } \\
\hline Model & Allied Telesis AT-MC102XL \\
\hline Wavelength & $1310 \mathrm{~nm}$ \\
\hline Maximum supported data rate & $100 \mathrm{Mbps}$ \\
\hline Data rates $(R)$ used & 1, $10 \mathrm{Mbps}$ \\
\hline \multicolumn{2}{|l|}{$\mathbf{T x}$} \\
\hline $\begin{array}{l}\text { Maximum optical power at } \\
\text { collimator }\end{array}$ & $23.4 \mu \mathrm{W}$ \\
\hline Fiber length $\&$ type & 1 meter - MM 50/125 \\
\hline Collimator & Optical microscope lens $25 \mathrm{x}$ \\
\hline Feedback fiber length \& type & 5 meters - MM 50/125 \\
\hline \multicolumn{2}{|l|}{ FSO channel } \\
\hline Channel length & $2.5 \mathrm{~m}$ \\
\hline $\begin{array}{l}\text { Distance between heating } \\
\text { elements }\end{array}$ & $7.5 \mathrm{~cm}$ \\
\hline Heating element length & $25 \mathrm{~cm}$ \\
\hline Fan speed & $\begin{array}{l}\text { I }->1400 \mathrm{rpm} \\
\text { II }->2800 \mathrm{rpm}\end{array}$ \\
\hline \multicolumn{2}{|l|}{$\mathbf{R x}$} \\
\hline Fiber length \& type & 1 meter - MM 50/125 \\
\hline Optical power after the Rx fiber & $5.8 \mu \mathrm{W}$ \\
\hline Focusing lens & Optical microscope lens $25 \mathrm{x}$ \\
\hline Photodetector type & GaAs \\
\hline Focusing lens active area & $0.38 \mathrm{~cm}^{2}$ \\
\hline DSO maximum BW & $350 \mathrm{MHz}$ \\
\hline DSO maximum sampling rate & $2 \mathrm{GSa} / \mathrm{s}$ \\
\hline
\end{tabular}

\section{Results and Discussions}

The results and discussion of our measurement campaign are outlined in this section. First, the system is tested with no turbulence as a baseline for two different values of $R(1$ and $10 \mathrm{Mbps})$ and then with turbulence for the same $R$. For each $R$, the file length was chosen to fit on a 20 seconds transmission window. The data collected by DSO was saved and used for determining $\sigma_{I}^{2}$ and $\sigma_{R}^{2}$ in order to evaluate the turbulence strength, as explained in previous sections. In addition, we compared the statistical distribution of the samples with the theoretically predicted GG distribution. Finally, in order to evaluate the packet loss due to turbulence, we automatically recorded, for each measurement, the number of lost packets and determined the system packet error rate (PER). To assess the improvement in the link quality, we compared the PER results with that obtained using RQCs (i.e. $P_{R R_{R Q}}$ ). 
Using the set-up, we were able to generate both weak and moderate turbulence regimes by turning on the heating elements and running the fan at specific speed (I, II) - see Table 1 - located near OC. In details, by increasing the air-flux, generated by the fan, we observed the decrease in the corresponding thermal gradient created along the OBPP and thus the turbulence condition. Stronger turbulence regimes can be created by increasing the distance between the fan and the OBPP as well as introducing more cold and hot air flows along the path. This is due to the fact that less air flux is blown towards the heating elements and thus the thermal gradient is higher. In this paper we will only illustrate the measurements performed for weak and moderate turbulence regimes, $\sigma_{R}^{2}<0.6$ due to the experimental limitations.

During the measurements, we used the attenuator to reduce the average signal received by MC2 and in particular we recorded a received power of about $2.5 \mu \mathrm{W}$ just before the beam splitter. In this way, we were able to record packet losses even under the weak turbulence regime and evaluate the performance of RQC. Using the developed software we could readily properly set the RaptorQ parameters, such as $L_{p}, K$ and $K_{r}$ according to channel conditions. We have investigated a range of values for $L_{p}$ and $K$ (200 to 1500) in order to obtain the best performances in terms of encoding/decoding speeds and of the amount of memory usage. In fact, for lower values of $L_{p}$ and $K$ (e.g., 100) the source file is split into a larger number of packets $[28,35]$. This results in increased volume of encoding/decoding processes, thus leading to reduced speed. On the other hand, higher $L_{p}$ and $K$ values (more than 5000) result in bigger source blocks, thus increasing the memory usage and reduced overall performance.

Herein, two different ranges of Rytov variance are analysed: 0.15-0.25 (weak) and 0.45-0.55 (moderate). In all the cases, the room temperature was kept at $23^{\circ} \mathrm{C}$. We show the results obtained for the weak and moderate turbulence regimes.

\section{a. RaptorQ codes for FSO under the weak turbulence regime}

In Table II, we compare the results obtained for the weak turbulence regime $0.15<\sigma_{R}^{2}<0.25$, a range of $R$ and RQCs parameters. The temperatures recorded using three thermometers, as well as $L_{p}, K$ and $K_{r}$ are also shown in the table. Note that for these measurements the overhead is kept either at $10 \%$ or $20 \%$. For each case we show the PER with no error

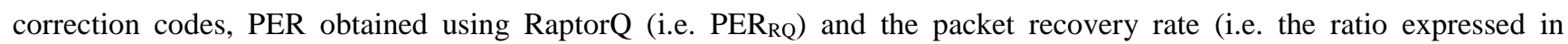
percentage between the number of packets recovered by RQCs and the number of lost packets). For the case 2 of Table II the signal fluctuation in the time domain and also both the predicted and the measured PDFs are shown in Fig. 2, for the weak turbulence regime with $\sigma_{I}^{2}=0.00765$ corresponding to $\sigma_{R}^{2}$ of 0.19 . Note that there is a good agreement between experimental and predicted PDF profiles, thus demonstrating the effectiveness of the practical set up. With regard to RQCs to ensure a $20 \%$ overhead we have used the following parameters: $L_{p}=200, K=200$, and $K_{r}=40$. The data rate, the number of packets transmitted and the number of packet lost are $1 \mathrm{Mbps}, 12480$, and 67 (i.e. $\sim 0.5 \%$ ), respectively. It is worth noting that using RQCs we were able to readily recover all the lost packets under the weak turbulence regime. 
TABle II - MEASUREMENT RESUlts: Rytov VARIANCE WITHIN THE RANGE $0.15 \div 0.25$

\begin{tabular}{ccccccccccccc}
\hline & $\begin{array}{c}\text { Data } \\
\text { rate } R \\
{[\mathrm{Mbps}]}\end{array}$ & $\begin{array}{c}L_{p} \\
{[\mathrm{byte}]}\end{array}$ & $K$ & $K_{r}$ & $\begin{array}{c}\mathrm{T} 1 \\
{\left[{ }^{\circ} \mathrm{C}\right]}\end{array}$ & $\begin{array}{c}\mathrm{T} 2 \\
{\left[{ }^{\circ} \mathrm{C}\right]}\end{array}$ & $\begin{array}{c}\mathrm{T} 3 \\
{\left[{ }^{\circ} \mathrm{C}\right]}\end{array}$ & $\sigma_{I}^{2}$ & $\sigma_{R}^{2}$ & PER & $\begin{array}{c}\text { PER } \\
\text { RQ }\end{array}$ & $\begin{array}{c}\text { Recovery } \\
\text { rate }[\%]\end{array}$ \\
\hline 1 & 1 & 200 & 200 & 20 & 32.2 & 27.4 & 35.6 & 0.00730 & 0.18 & $2.80 \times 10^{-3}$ & $1.75 \times 10^{-3}$ & 37.5 \\
2 & 1 & 200 & 200 & 40 & 31.0 & 26.7 & 34.0 & 0.00765 & 0.19 & $5.37 \times 10^{-3}$ & 0 & 100 \\
3 & 10 & 200 & 200 & 20 & 33.7 & 26.9 & 35.7 & 0.00832 & 0.21 & $1.61 \times 10^{-3}$ & $1.30 \times 10^{-3}$ & 19.0 \\
4 & 10 & 200 & 200 & 40 & 34.0 & 27.0 & 34.0 & 0.00747 & 0.19 & $3.97 \times 10^{-3}$ & $3.18 \times 10^{-3}$ & 19.9 \\
5 & 1 & 500 & 500 & 50 & 35.0 & 27.3 & 34.8 & 0.00783 & 0.20 & $2.50 \times 10^{-3}$ & 0 & 100 \\
6 & 1 & 500 & 500 & 100 & 35.0 & 27.0 & 34.0 & 0.00663 & 0.16 & $4.79 \times 10^{-3}$ & 0 & 100 \\
7 & 10 & 500 & 500 & 50 & 31.7 & 27.0 & 34.4 & 0.00768 & 0.19 & $1.64 \times 10^{-3}$ & 0 & 100 \\
8 & 10 & 500 & 500 & 100 & 31.0 & 26.0 & 33.0 & 0.00764 & 0.19 & $2.23 \times 10^{-3}$ & 0 & 100 \\
9 & 1 & 1000 & 1000 & 100 & 35.6 & 27.9 & 36.0 & 0.00820 & 0.21 & $1.36 \times 10^{-3}$ & 0 & 100 \\
10 & 1 & 1000 & 1000 & 200 & 34.4 & 38.0 & 34.0 & 0.00830 & 0.21 & $6.25 \times 10^{-3}$ & 0 & 100 \\
11 & 10 & 1000 & 1000 & 100 & 32.0 & 26.7 & 33.4 & 0.00734 & 0.18 & $3.27 \times 10^{-3}$ & 0 & 100 \\
12 & 10 & 1000 & 1000 & 200 & 33.6 & 27.3 & 33.6 & 0.00753 & 0.19 & $6.58 \times 10^{-3}$ & 0 & 100 \\
13 & 1 & 1500 & 1500 & 150 & 32.1 & 26.9 & 34.5 & 0.00859 & 0.22 & $1.09 \times 10^{-2}$ & 0 & 100 \\
14 & 1 & 1500 & 1500 & 300 & 33.9 & 27.0 & 33.9 & 0.00832 & 0.21 & $6.67 \times 10^{-2}$ & 0 & 100 \\
15 & 10 & 1500 & 1500 & 150 & 33.5 & 27.8 & 34.6 & 0.00828 & 0.21 & $4.04 \times 10^{-3}$ & 0 & 100 \\
16 & 10 & 1500 & 1500 & 300 & 36.0 & 27.4 & 34.0 & 0.00697 & 0.17 & $5.80 \times 10^{-3}$ & 0 & 100 \\
\hline
\end{tabular}
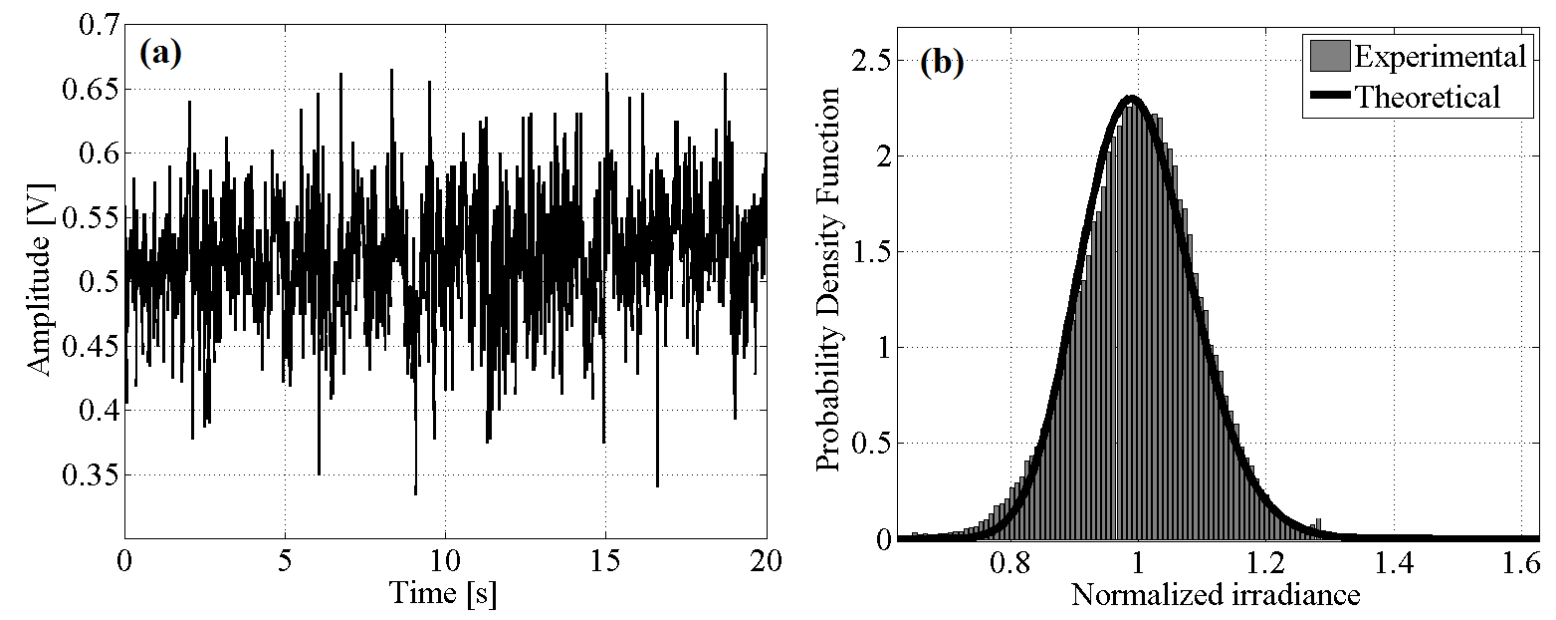

FIG. 2. Illustration of generated weak turbulence $\left(\sigma_{I}^{2}=0.00765, \sigma_{R}^{2}=0.19\right)$ : (a) signal fluctuations, and (b) predicted and measured probability density functions.

As shown in Table II, the recovery rate increases both with the overhead (e.g., comparing Cases 1 and 2 ) and with $L_{p}$ and $K$, reaching $100 \%$ for $L_{p}=K=500$ for both values of overhead and data rates under the weak turbulence regime. Note that for $L_{p}=K=200$ we observe only an overall lower improvement in the link performance. This is because at lower values of $L_{p}$ and $K$ a greater number of smaller packets is generated and transmitted in the same time interval. Moreover, when using this configuration, it is possible to lose only up to 20 packets (for an overhead of $10 \%$ ) or 40 packets (for an overhead of $20 \%$ ) for every transmitted matrix. Since more than 20 packets are lost as a result of fading under the aforementioned turbulence conditions, then it is not recommended to use this configuration. This problem can be resolved 
by means of increasing further the redundancy, but at the cost of reduced system throughput. However, the best option to increase the recovery rate and maintain the throughput level would be to vary both the packet length and the number of source packets, while keeping the overhead constant.

In addition, see Table II, we emphasize that for configurations with low data recovery decreasing $R$ represents a possible solution to improve the recovery rate. This can be easily explained for the case of fixed fading condition, where the number of consecutive lost packets diminishes with decreasing $R$, thus increasing the packet recovery rate. For example, for Cases $1 \& 3$ the recovery rate decreases from $38 \%$ to $19.0 \%$ when $R$ is increased from 1 to 10 Mbps. In the same way, for Case 2 at $R$ of $1 \mathrm{Mbps}$, all the lost packets were successfully recovered, whereas for Case 4 at $R$ of $10 \mathrm{Mbps}$ the recovery rate was only $\sim 20 \%$.

\section{b. RaptorQ codes for FSO under the moderate turbulence regime}

Next we considered the moderate turbulence condition. This was achieved by setting the speed of the fan to the minimum value (I). In Table III, we display the results obtained for moderate turbulence regimes for a range of RQCs parameters and $R$. In particular, we show the results for Rytov variances values within the range $0.45 \div 0.55$.

TABLE III - MEASUREMENT RESUlTS: Rytov VARIANCE WITHIN THE RANGE $0.45 \div 0.55$

\begin{tabular}{ccccccccccccc}
\hline & $\begin{array}{c}\text { Data } \\
\text { rate } R \\
{[\mathrm{Mbps}]}\end{array}$ & $\begin{array}{c}L_{p} \\
{[\mathrm{byte}]}\end{array}$ & $K$ & $K_{r}$ & $\begin{array}{c}\mathrm{T} 1 \\
{\left[{ }^{\circ} \mathrm{C}\right]}\end{array}$ & $\begin{array}{c}\mathrm{T} 2 \\
{\left[{ }^{\circ} \mathrm{C}\right]}\end{array}$ & $\begin{array}{c}\mathrm{T} 3 \\
{\left[{ }^{\circ} \mathrm{C}\right]}\end{array}$ & $\sigma_{I}^{2}$ & $\sigma_{R}^{2}$ & PER & PER ${ }_{\text {RQ }}$ & $\begin{array}{c}\text { Recovery } \\
\text { rate }[\%]\end{array}$ \\
\hline 1 & 1 & 200 & 200 & 20 & 28.7 & 24.8 & 37.1 & 0.01863 & 0.55 & $1.49 \times 10^{-2}$ & $1.27 \times 10^{-2}$ & 15.2 \\
2 & 1 & 200 & 200 & 40 & 34.5 & 28.2 & 39.3 & 0.01829 & 0.55 & $6.97 \times 10^{-3}$ & 0 & 100.0 \\
3 & 10 & 200 & 200 & 20 & 28.7 & 24.8 & 36.2 & 0.01643 & 0.47 & $3.70 \times 10^{-3}$ & $1.76 \times 10^{-3}$ & 52.5 \\
4 & 10 & 200 & 200 & 40 & 37.2 & 27.5 & 28.9 & 0.01783 & 0.53 & $1.11 \times 10^{-2}$ & $7.62 \times 10^{-3}$ & 31.1 \\
5 & 1 & 500 & 500 & 50 & 38.7 & 24.7 & 36.2 & 0.01614 & 0.46 & $1.59 \times 10^{-2}$ & 0 & 100.0 \\
6 & 1 & 500 & 500 & 100 & 30.4 & 26.4 & 39.0 & 0.01777 & 0.52 & $1.08 \times 10^{-2}$ & 0 & 100.0 \\
7 & 10 & 500 & 500 & 50 & 28.9 & 25.3 & 38.1 & 0.01862 & 0.55 & $1.97 \times 10^{-2}$ & $1.43 \times 10^{-2}$ & 27.4 \\
8 & 10 & 500 & 500 & 100 & 30.3 & 26.7 & 39.9 & 0.01778 & 0.53 & $7.65 \times 10^{-3}$ & 0 & 100.0 \\
9 & 1 & 1000 & 1000 & 100 & 28.8 & 25 & 39.4 & 0.01651 & 0.48 & $2.41 \times 10^{-2}$ & 0 & 100.0 \\
10 & 1 & 1000 & 1000 & 200 & 29.6 & 26.7 & 37.4 & 0.01787 & 0.53 & $1.33 \times 10^{-2}$ & 0 & 100.0 \\
11 & 10 & 1000 & 1000 & 100 & 31.0 & 26.6 & 40.0 & 0.01609 & 0.46 & $1.95 \times 10^{-2}$ & $6.77 \times 10^{-3}$ & 65.2 \\
12 & 10 & 1000 & 1000 & 200 & 29.0 & 25.4 & 37.8 & 0.01602 & 0.46 & $6.63 \times 10^{-3}$ & 0 & 100.0 \\
13 & 1 & 1500 & 1500 & 150 & 28.9 & 25.0 & 38.0 & 0.01713 & 0.50 & $1.82 \times 10^{-2}$ & 0 & 100.0 \\
14 & 1 & 1500 & 1500 & 300 & 30.5 & 24.9 & 30.5 & 0.01595 & 0.46 & $1.39 \times 10^{-2}$ & 0 & 100.0 \\
15 & 10 & 1500 & 1500 & 150 & 29.5 & 24.7 & 36.2 & 0.01691 & 0.49 & $2.13 \times 10^{-2}$ & 0 & 100.0 \\
16 & 10 & 1500 & 1500 & 300 & 28.8 & 25.3 & 37.1 & 0.01718 & 0.50 & $1.62 \times 10^{-2}$ & 0 & 100.0 \\
\hline
\end{tabular}

For the Case 4 of the Table III, figure 3 depicts the measured signal fluctuations and predicted and measured PDF profiles corresponding to $\sigma_{I}^{2}=0.01783$ and $\sigma_{R}^{2}=0.53$. Here, for RQCs we selected $L_{p}=K=200, K_{r}=40$ (again, an 
overhead of $20 \%$ ) and $R=10 \mathrm{Mbps}$. We transmitted a total of $124.8 \mathrm{k}$ packets and recorded 1381 lost packets (i.e. $~ 1.1 \%$ ) of which 430 were recovered because of RQCs. Hence, the PER decreased from $1.11 \times 10^{-2}$ to $7.62 \times 10^{-3}$, thus demonstrating the capabilities of RQCs as a powerful fading mitigation technique even under moderate turbulence regimes. Moreover, it is worth noting that the errors are not uniformly distributed over the entire recorded interval but, as expected, they occur whenever the detected signal drops below the receiver threshold because of fading. For example, in Fig. 3(a), high packet losses occurred in the time intervals of 7, 11, 13 and 16 seconds, but not between 8 and 9 seconds.
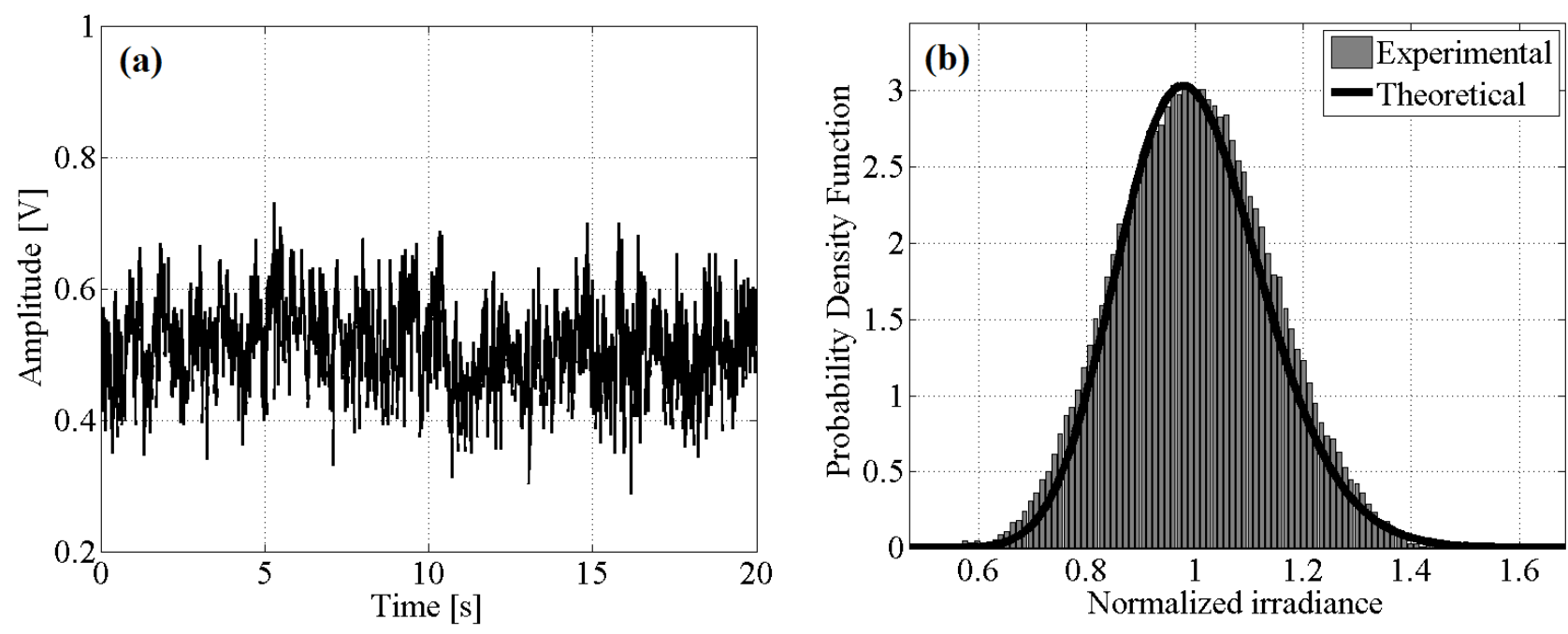

FIG. 3. Example of generated moderate turbulence ( $\sigma_{I}^{2}=0.01783, \sigma_{R}^{2}=0.53$ ): (a) signal fluctuations; (b) measured and predicted probability density functions.

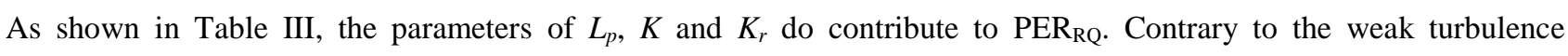
condition, for $L_{p}=K=500$ complete data recovery for a $10 \%$ overhead was not possible. As reported in the table, for

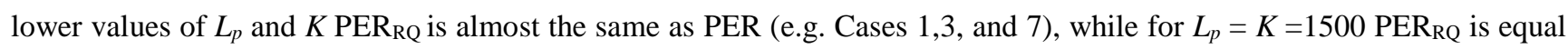

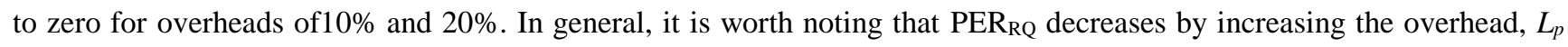
and $K$, while increases with $R$. Hence, we can conclude that RQCs represent a versatile fading mitigation technique for reducing the data packet loss in FSO communications as a result of turbulence. Based on practical measurements carried out using the proposed experimental set up, the main strength of RQCs resides in the ability to change encoding and decoding parameters according to the channel conditions, in order to reduce the packet loss. Moreover, we highlighted that, for the cases with a recovery rate $<100 \%$, the system performances could be further improved by increasing the redundancy.

\section{Conclusions}

In this work, we reported on an experimental set-up to assess the FSO link performance under weak to moderate turbulence regimes (i.e. Rytov variance up to 0.6). We considered different link conditions and using a data post- 
processing technique the optical turbulence strength was determined by calculating the scintillation index and the Rytov variance. We showed that there is a good agreement between predicted and measured PDFs.

In addition, we presented the results in terms of packet losses due to erasure errors for the FSO link for a range of turbulence regimes. An innovative data transfer solution that integrated the most recent rateless codes was implemented and tested in order to reduce or even completely eliminate the erasure errors and packet errors. The aforementioned results showed that RQC can lead to lower PER. Finally, the obtained results demonstrated the potential of RQ as a fading mitigation technique in FSO communications to improve the link performance by reducing effects of turbulence induced erasure errors.

\section{Acknowledgements}

This work was supported by the European Space Agency under grant no. 5401001020 . We are very grateful to Dr. E. Armandillo for enlightening discussions. This research project also falls within the frame of COST ICT Action IC1101 Optical Wireless Communications - An Emerging Technology (OPTICWISE). J. Perez's work is supported by Spanish MINECO Juan de la Cierva JCI-2012-14805.

\section{References}

[1] Ghassemlooy, Z., Popoola, W., Rajbhandari, S.: 'Optical Wireless Communications: System and Channel Modelling with MATLAB’ (CRC Press, Boca Raton, FL, 2012)

[2] Tsukamoto, K., Hashimoto, A., Aburakawa, Y., Matsumoto, M.: 'The case for free space', IEEE Microw. Mag., 2009, 10, (5), pp. 84-92

[3] Paraskevopoulos, A., Vučić, J. V, Voss, S.-H., Swoboda, R., Langer, K.-D.: 'Optical wireless communication systems in the Mb/s to Gb/s range, suitable for industrial applications', IEEE/ASME Trans. Mechatronics, 2010, 15, (4), pp. 541-547

[4] Ghassemlooy, Z., Le Minh, H., Rajbhandari, S., Perez, J., Ijaz, M.: 'Performance analysis of Ethernet/fast-Ethernet free space optical communications in a Controlled Weak Turbulence Condition', J. Lightw. Technol., 2012, 30, (13), pp. 2188-2194

[5] Ciaramella, E., Arimoto, Y., Contestabile, G., Presi, M., D’Errico, A., Guarino, V., Matsumoto, M.: ‘ $1.28-\mathrm{Tb} / \mathrm{s}$ (32 × 40 Gb/s) free-space optical WDM transmission system', IEEE Photon. Technol. Lett., 2009, 21, (16), pp. 1121-1123

[6] Parca, G., Shahpari, A., Carrozzo, V., Tosi Beleffi, G. M. T., Teixeira, A. L. J.: '1.6 Terabit/s (16x100 Gigabit/s) optical wireless transmission for next generation convergent urban infrastructures', Opt. Eng., 2013, 52, (11), pp. 116102

[7] Leitgeb, E., Gebhart, M., Birnbacher, U., Sheikh Muhammad, S., Chlestil, C.: 'Applications of free space optics for broadband access', in OpNeTec, volume 164 of IFIP International Federation for Information Processing, pp. 579-586: Springer, 2004 
[8] Leitgeb, E., Awan, M.S., Plank, T., Perlot, N., Capsoni, C., Nebuloni, R., Javornik, T., Kandus, G., Nadeem, F., Brandl, P., Muhammad, S.S., Loschnigg, M., Khan, M.S., Duca, E., Betti, S.: 'Investigations on free-space optical links within SatNEx II'. Proc. 3rd European Conf. on Antennas and Propagation (EuCAP 2009), Berlin, Germany, 23-27 March 2009, pp. 1707-1711

[9] Mori, S., Marzano, F.S., Frezza, F., Beleffi, G.M.T., Carrozzo, V., Busacca, A., Ando, A.: 'Model analysis of hydrometeor scattering effects on free space near-infrared links'. Proc. Int. Workshop on Optical Wireless Communications (IWOW 2012), Pisa, Italy, 22 October 2012, pp.1-3

[10] Andrews, L. C., Phillips, R. L., Hopen, C. Y.: 'Laser Beam Scintillation with Applications' (SPIE Press, Bellingham, WA, 2001)

[11] Andrews, L. C., Phillips, R. L., Hopen, C. Y.: 'Laser Beam Propagation through Random Media' (SPIE Press, Bellingham, WA, 2005, 2nd edn.)

[12] Hulea, M., Ghassemlooy, Z., Rajbhandari, S., Xuan Tang,: 'Compensating for Optical Beam Scattering and Wandering in FSO Communications,' J. Lightwave Technol., 2014, 32, (7) , pp.1323-1328

[13] Ghassemlooy, Z., Popoola, W. O., Ahmadi, V., Leitgeb, E.: 'MIMO free-space optical communication employing subcarrier intensity modulation in atmospheric turbulence channels', in Communications Infrastructure. Systems and Applications in Europe, vol. 16, part 2, pp. 61-73, Berlin Heidelberg: Springer, 2009

[14] Weerackody, V., Hammons, A. R., Tebben, D. J.: 'Multi-input multi-output free space optical satellite communication links', Proc. 41st Ann. Conf. Inform. Sci. Sys. (CISS '07), 2007, pp. 679-683

[15] Zhao, Z., Lyke, S., Roggemann, M.: “Adaptive optical communication through turbulent atmospheric channels,” Proc. IEEE Int. Conf. on Communications, 2008, pp. 5432-5436

[16] Zhijun Zhao, Rui Liao, Lyke, S.D., Roggemann, M.C.: 'Reed-Solomon coding for free-space optical communications through turbulent atmosphere,' 2010 IEEE Aerospace Conf, , 6-13 March 2010, pp.1-12

[17] Garcia-Zambrana, A.: 'Error rate performance for STBC in free-space optical communications through strong atmospheric turbulence,' IEEE Commun. Lett., 2007, 11, pp. 390-392

[18] Abou-Rjeily, C.: 'On the optimality of the selection transmit diversity for MIMO-FSO links with feedback,' IEEE Commun. Lett., 2011, 15, (6), pp. $641-643$

[19] García-Zambrana, A., Castillo-Vázquez, C., Castillo-Vázquez, B.: 'Rate-adaptive FSO links over atmospheric turbulence channels by jointly using repetition coding and silence periods', Opt. Exp., 2010, 18, (24), pp. 25422-25440

[20] Andò, A., Mangione, S., Curcio, L., Stivala, S., Garbo, G., Pernice, R., Busacca, A.C.: 'Recovery capabilities of rateless codes on simulated turbulent terrestrial free space optics channel model', Int. J. Antennas Propag., 2013, Volume 2013, Article ID 692915, 8 pages

[21] Luby, M.: 'LT codes'. Proc. 43rd Annual IEEE Symposium on Foundations of Computer Science, Vancouver, Canada, 16-19 November 2002, pp. 271-280

[22] MacKay, D. J. C.: 'Fountain codes’, IEE P-Commun., 2005, 152, (6), pp. 1062-1068

[23] Shokrollahi, A.: 'Raptor codes', IEEE Trans. Inf. Theory, 2006, 52, (6), pp. 2551-2567

[24] Demir, U., Aktas, O.: 'Raptor versus Reed Solomon forward error correction codes'. Proc. 2006 Int. Symp. on Computer Networks, 2006, pp. 264-269 
[25] Bouras, C., Kanakis, N., Kokkinos, V., Papazois, A.: 'Enhancing reliable mobile multicasting with RaptorQ FEC', Proc. 2012 IEEE Symp. on Comp. and Comm., 2012, pp. 82-87

[26] Luby, M., Shokrollahi, A., Watson, M., Stockhammer, T., Minder, L.: 'RaptorQ Forward Error Correction Scheme for Object Delivery', RFC, 6330_-Proposed Standard, IETF, 2007

[27] QUALCOMM, 'RaptorQ technical overview,” tech. rep., United States, 2010

[28] Pernice, R., Andò, A, Musso, D., Parisi, A, Mangione, S., Curcio, L., Stivala, S., Busacca, A.C., 'Packet loss recovery in an indoor Free Space Optics link using rateless codes', 16th Int. Conf. on Transparent Optical Networks (ICTON 2014), 2014, pp.1-4

[29] Pernice, R., Andò, A, Musso, D., Lionti, F., Parisi, A, Mangione, S., Adamo, G., Curcio, L., Stivala, S., Busacca, A.C., 'Rateless codes mitigation technique in a turbulent indoor Free Space Optics link', 2014 Third Mediterranean Photonics Conference, 2014, pp.1-3

[30] Anguita, J. A., Neifeld, M. A., Hildner, B., Vasic, B.: 'Rateless coding on experimental temporally correlated fso channels', J. Lightw. Technol., 2010, 28, (7), pp. 990-1002

[31] Wang, N., Cheng, J.: 'Moment-based estimation for the shape parameters of the Gamma-Gamma atmospheric turbulence model', Opt. Exp., 2010, 18, (12), pp. 12824-12831

[32] Mudge, K. A., Dilusha Silva, K. K. M. B, Clare, B. A., Grant, K. J., Nener, B. D.: 'Scintillation index of the free space optical channel: Phase screen modelling and experimental results', Proc. 2011 Int. Conf. on Space Optical Systems and Applications (ICSOS), 2011, pp. 403-409.

[33] Zvanovec, S., Perez, J., Ghassemlooy, Z., Rajbhandari, S., Libich, J.: 'Route diversity analyses for free-space optical wireless links within turbulent scenarios', Opt. Exp., 2013, 21, (6), pp. 7641-7650

[34] Pernice, R., Andò, A., Cardinale, M., Curcio, L., Stivala, S., Parisi, A., Busacca, A.C., Ghassemlooy, Z., Perez, J.: 'An indoor FSO link under the weak turbulence regime: measurements and model validation', IET Commun., 2015, 9, (1), pp. 62-70

[35] Pernice, R., Andò, A., Curcio, L., et al.: 'Statistical analysis of RaptorQ failure probability applied to a data recovery software'. 2014 Euro Medi Telco Conf. (EMTC), 2014, pp. 1-5 